consequent difficulty of breathing, about a month before the peculiar coldness of the feet was noticed.

Duchenne states briefly - "Some patients accuse sudden or too prolonged cooling, suppression of transpiration ; thus, for example, there is a sportsman who often has remained a long time with the feet and legs in the water. Once, the phenomena of this disease have shown themselves after a too prolonged sitz bath. Trousseau mentions hereditary taint, also, as a predisposing causc. This may appear in the first case, where it is stated that the father died of paralysis.

Out of 132 cases referred to by Carré, some of which, however, were not of this disease, 66 have no cause assigned, and no anteccdents are given. In 17 there was a previous history of syphilis, in 8 of rheumatism, 6 had been exposed to unusual and frequent fatigue, 23 had been subjected to cold and dampness in various ways-either dwelling in damp houses, sleeping in the open air on damp ground, working in damp places, or were subject to sudden cooling while perspiring freely, and immediately after felt the first signs of the disease. In 9 , there had been emotional excitement or long-continued anxiety. In 5 , disease of the nervous system had existed among the ancestors. In 5 , abuse of wine or venereal excess had been noted.

[To be concluded.]

\section{RUPTURE OF THE UTERUS AND VAGINA.}

By Edward Barton, M.D., of Orange, Mass.

I was called, April 7th, 7 o'clock, A.M., to Mrs. - Irish, aged 40 , mother of three children, one stillborn. She was of medium size, rather fleshy, of uniform good health, except that she had a bronchial difficulty, with hoarseness. I learned from two fenale attendants and the patient, that she was attacked with labor pains about 5,P.M., the day before; that the full time for labor had arrived ; that labor progressed apparently favorably till $10 \frac{1}{2}$ o'clock, when she had a very severe pain, accompanied with a tearing sensation low down in the left side, and an audible snap, which was heard in an adjoining room; that the pains ceased from that time; that severe hæmorrhage and free vomiting ensued, and that she became cold and languid. I found her with pale and sunken countenance, cold extremities and fceble pulse, and occasionally eructating dark-colored matter from the sto- mach, which she frequently had done during the night.

Under these circumstances, I made a vaginal examination, but finding such a confused state of things that I could not ascertain anything satisfactorily by the feel of the finger, I carried the hand within the vagina and discovered a laceration, and an entire separation of the vagina from the uterus, with two longitudinal rents in the upper third of the vagina-one anterior, the other posterior-and both a little to the left of the median line; these rents extending into the left portion of the cervix uteri, and there meeting. This triangular formed flap was turned down and pendulous within the vagina, some three inches. The uterus was high up, its cervix resting to the right of the sacro-vertebral prominence, with its fundus turned to the right, and apparently empty and firmly contracted. The foetus was not within reach while the hand was within the pelvis, but it could be felt through the abdominal parietes, above the umbilicus.

I could not satisfy myself whether the membranes had been ruptured before the accident, but the patient was sure that there had been no sudden gush of water.

It appeared very clear that the fœtus and placenta had escaped within the abdominal cavity, and I explained the nature of the case to the patient, and informed her of what the certain result must he. Under the circumstances, it seemed a serious question whether it would be an act of mercy to attempt to do anything with a view of delivery.

She was stimulated, had warmth applied to the extremities, and an opiate administered; after the lapse of several hours, the patient not getting materially worse, I decided to make an attempt to deliver. She was etherized; the hand, moistened with warm water, was carried through the rent to the left of the uterus, its fundus grasped within the hand, and found empty and firmly contracted. The hand passed up, with its dorsum to the parietes, above the umbilicus, and rested upon the brecch of the foetus, which lay transversely, with its head and shoulders in the left iliac region; but the omentum intervened, and the hand was drawn down and passed beneath the omen. tum, the breech gained, and then by degrees the right foot. Failing to secure tile left, the right was drawn down and secured, and then the left found and brought down also; traction was commenced, and after some difficulty the patient was delivered of a large female child, and then of 
the placenta, with the cord entire. The hand was again passed within the vagina; there was no protrusion of the viscera through the rent, and the lacerated parts were adjusted as well as circumstances would admit.

At this stage of proceeding, the patient was very low, the pulse being hardly perceptible. As soon as she could be made to swallow, stimulants were freely given, warm applications to the extremities and warm spirit lotion to the abdomen. At the end of an hour reaction had slightly taken place, and at the expiration of five hours she appeared about as well as before I commenced delivery. At 8 o'clock, P.M., her pulse was 140 , and I left her with morphine gr. \& every two hours, to be alternated with quinine gr. $\frac{1}{6}$, with whiskey at short intervals.

8th, A.M.-Patient slept two hours during the night. Has vomited frequently; is thirsty; tongue dry; has better warmth of extremities ; complains of some abdominal tenderness, but no pain; abdomen rather full, but not tympanitic; has urinated; no hæmorrhage ; countenance full as good; pulse 140. Continue the medicine. Has crust tea.

P.M.-No worse. Continue treatment.

9th.-Slept considerable during the night. Says she "feels no way worse." Skin is moist and warm; vomits the same dark fluid, and is thirsty ; pulse 136 ; abdominal tenderness about the same. Continue the treatment.

P.M.-Has passed the day very well. Occasionally vomits; no vaginal discharge. No change of treatment.

10th.-Slept during the night. Skin is warm and moist; vomits the same dark fluid; is thirsty; tongue dry and covered with brownish coat; abdomen is tympanitic ; no increase of tenderness; no pain; pulse 135. For the first time, there is a discharge from the vagina of bloody serum, which ran through the bed in a puddle on the floor.

11th.-No particular change. Vomits; less vaginal discharge; pulse 132 .

12th.-There is no great change. Has very little pain or tenderness; vomits, and is thirsty; pulse 136. Up to this time, there has been no change in the breasts, but now there is some hardness and she feels chilly.

13th.-Did not pass a good night, but there is no change in appearances. Pulse 135. There is secretion of milk; urinates well ; foetid vaginal discharge.

14 th.-Bowels moved freely by enema; pulse 132; no secretion of milk.
15th.-Is as well. Vomits less; has less tenderness; bowels moved voluntarily; pulse 132 .

16th.-Did not pass a good night; was restless. Complains of pain in umbilical region; the navel pouches out, and there is increase of heat and tumefaction of the tissues about the region. Pulse 132. The discharge from the vagina is described as looking like yolk of egg, and is very fotid. There is more dryness of the mouth, and thirst. Omit quinine.

19th.-Aphthous deposition on tongue.

21 st.-Appears quite as well ; very little tenderness; bowels soft and less full ; no discharge from vagina; pulse 115 .

24 th. - Sat on the side of the bed yesterday more than an hour. Did not rest as well last night; has less appetite ; vomited in the night; pulse 120 .

25th.-Seems better. Tongue moist and clean ; takes more nourishment; pulse 90 ; bowels soft; no vaginal discharge. Diminish morphine one half.

27th.-Bowels moved by enema, and in twelve hours moved twice voluntarily, and vomited; pulse 95.

29th.-No nausea; no thirst; no tenderness; bowels soft; no vaginal discharge; pulse 102 .

At 4 o'clock, A.M., on May 5th, she had a chill, with pain in her bowels, her extremities became cold, and she died in one hour, having lived twenty-eight days and six hours after the accident. No autopsy.

I made no vaginal cxamination after the day of delivery.

In treating the case, my olject from the commencement was to keep her constantly under the influence of opiates, not to the extent of narcotism, but so that its effects could be felt by the patient and scen by others. The patient was not well nursed.

She was allowed to change her position at pleasure, and she turned from side to side, and had strength to help herself considerably, and for the last few days prior to the last $t w o$, there was an increase of strength. Her bowels being so natural, soft, and free from tenderness, and the vaginal discharge having ceased for so long a time, seemed to indicate that she was doing well ; and at times, losing sight of my better judgment, I would half believe that sho would recover.

It is worthy of mention that the patient had a large sore on the nates, apparently the result of a scald from hot applications during labor, by the fernale attendants. What infuence, if any, this had in producing rupture, we cannot decide. 